\title{
Les résultats des élèves asiatiques dans les enquêtes internationales
}

Asian pupils' results in international surveys

Los resultados de los alumnos asiáticos en las encuestas internacionales

Jean-Marie De Ketele

\section{OpenEdition}

Journals

Édition électronique

URL : https://journals.openedition.org/ries/4351

DOI : 10.4000/ries.4351

ISSN : 2261-4265

Éditeur

France Education international

Édition imprimée

Date de publication : 4 avril 2015

Pagination : 69-80

ISBN : 9782854206067

ISSN : 1254-4590

\section{Référence électronique}

Jean-Marie De Ketele, "Les résultats des élèves asiatiques dans les enquêtes internationales », Revue internationale d'éducation de Sèvres [En ligne], 68 | avril 2015, mis en ligne le 04 avril 2017, consulté le 01 juillet 2021. URL : http://journals.openedition.org/ries/4351 ; DOI : https://doi.org/10.4000/ries. 4351

(c) Tous droits réservés 


\title{
Les résultats des élèves asiatiques dans les enquêtes internationales
}

\author{
Jean-Marie De Ketele \\ Université catholique de Louvain, Belgique
}

Les résultats des élèves asiatiques dans les enquêtes internationales ${ }^{1}$, principalement PISA, ont été largement médiatisés et ont interrogé tous ceux qui s'intéressent à l'éducation et aux secteurs qui lui sont reliés. De nombreuses questions sont posées : comment les résultats se positionnent-ils par rapport aux pays occidentaux ? Se diversifient-ils selon les pays et les territoires ? Parviennentils à allier efficacité (rapport entre les résultats observés et les résultats attendus) et équité (distribution juste à travers les différentes catégories de population)? Sont-ils associés à certaines caractéristiques sociales, économiques et culturelles des régions concernées? Quelle confiance peut-on accorder aux résultats des enquêtes internationales ? Leur médiatisation n'occulte-t-elle pas d'autres enjeux non pris en considération? Peut-on se contenter des performances des élèves pour évaluer la qualité des systèmes éducatifs asiatiques? Mais aussi en quoi ces résultats invitent-ils nos systèmes éducatifs occidentaux à s'interroger ?

Si toutes ces questions sont régulièrement posées, les réponses données relèvent encore trop souvent du domaine de l'opinion ou restent encore trop souvent à la surface visible des informations rapportées. Cet article a donc pour ambition d'analyser les résultats disponibles des élèves asiatiques dans les enquêtes internationales PISA sous un double aspect : leurs faces visibles et leurs faces moins visibles (souvent encore occultées, voire cachées).

Nous sommes conscient des limites de notre analyse. La première est objective : les résultats des élèves asiatiques dans les enquêtes internationales ne concernent pas tous les pays et territoires asiatiques; il faut donc éviter les généralisations abusives. La seconde est davantage subjective : malgré notre volonté de décentration, notre regard reste malgré tout occidental.

Notre raisonnement suivra quatre étapes : les deux premières porteront sur les faces les plus directement visibles (l'analyse des résultats disponibles, puis l'analyse des covariations entre ces résultats et une série d'indicateurs

1. Cet article s'appuie sur une conférence prononcée par l'auteur le 12 juin 2014 au Centre international d'études pédagogiques, à Sèvres, lors du colloque international "L'éducation en Asie en 2014 : quels enjeux mondiaux ? " (12-14 juin 2014) organisé à l'occasion du $20^{\mathrm{e}}$ anniversaire de la Revue internationale d'éducation de Sèvres. (NdIR) Voir la vidéo de cette conférence : http://goo.gl/ $\mathrm{X}_{5} \mathrm{nRrX}$; pour en savoir plus sur le colloque : http://ries.revues. org/3710 (NdIR) 
objectifs) et les deux autres sur des faces moins visibles (les aspects occultés des enquêtes internationales; les faces peu exploitées ou peu connues de la qualité des systèmes éducatifs asiatiques).

\section{LES FACES VISIBLES : LES RÉSULTATS EN TERMES D'EFFICACITÉ ET D'ÉQUITÉ}

Nous avons rassemblé dans le tableau 1 trois types de résultats particulièrement importants, issus de l'enquête PISA 2012 : les scores moyens, obtenus aux trois épreuves de culture mathématique, de lecture et de science, donnent une mesure globale de l'efficacité des systèmes éducatifs dans ces domaines; les pourcentages d'élèves peu performants (sous le niveau 2) et très performants (niveau 5 ou plus) permettent d'apprécier l'une des facettes importantes de l'équité des systèmes. Le tableau 1 concerne les onze pays ou territoires asiatiques ayant participé à PISA 2012, la moyenne des pays de l'OCDE, deux pays (la Finlande et le Canada) souvent considérés en Occident comme des pays de référence, et la France qui fut le pays hôte du colloque international «L'éducation en Asie en 2014 : quels enjeux mondiaux?».

Tableau 1.

Les résultats aux épreuves PISA 2012

\begin{tabular}{|l|c|c|c|c|c|c|c|c|c|}
\hline \multirow{2}{*}{ Pays } & \multicolumn{3}{|c|}{ Scores moyens } & \multicolumn{3}{c|}{$\begin{array}{c}\text { \% d'élèves } \\
\text { peu performants }\end{array}$} & \multicolumn{3}{c|}{$\begin{array}{c}\text { \% d'élèves } \\
\text { très performants }\end{array}$} \\
\cline { 2 - 12 } & Math. & Lect. & Science & Math. & Lect. & Science & Math. & Lect. & Science \\
\hline Shanghai & 613 & 570 & 580 & 3,8 & 2,9 & 2,7 & 55,4 & 25,1 & 27,2 \\
\hline Singapour & 573 & 542 & 551 & 8,3 & 5,9 & 9,6 & 40,0 & 21,2 & 22,7 \\
\hline Hong-Kong & 561 & 545 & 555 & 8,5 & 6,8 & 5,6 & 33,7 & 16,8 & 16,7 \\
\hline Taïwan & 560 & 523 & 523 & 12,8 & 11,5 & 9,8 & 37,2 & 11,8 & 8,3 \\
\hline Corée du Sud & 554 & 536 & 538 & 9,1 & 7,6 & 6,6 & 30,9 & 14,1 & 11,7 \\
\hline Macao & 538 & 509 & 521 & 10,8 & 11,5 & 8,8 & 24,3 & 7,0 & 6,7 \\
\hline Japon & 536 & 538 & 547 & 11,1 & 9,8 & 8,5 & 23,7 & 18,5 & 18,2 \\
\hline Vietnam & 511 & 508 & 528 & 14,2 & 9,4 & 6,7 & 13,3 & 4,6 & 8,1 \\
\hline Thaïlande & 427 & 441 & 499 & 49,7 & 33,0 & 33,6 & 2,6 & 0,8 & 0,9 \\
\hline Malaisie & 421 & 398 & 420 & 51,8 & 52,7 & 45,5 & 1,3 & 0,1 & 0,3 \\
\hline Indonésie & 375 & 396 & 382 & 75,7 & 55,2 & 66,6 & 0,3 & 0,1 & 0,0 \\
\hline Moyenne OCDE & 494 & 496 & $\mathbf{5 0 1}$ & $\mathbf{2 3 , 0}$ & $\mathbf{1 8 , 0}$ & $\mathbf{1 7 , 8}$ & $\mathbf{1 3 , 1}$ & $\mathbf{8 , 4}$ & $\mathbf{8 , 3}$ \\
\hline Finlande & 519 & 524 & 545 & 12,3 & 11,3 & 7,7 & 15,3 & 13,5 & 17,1 \\
\hline Canada & 518 & 523 & 525 & 13,8 & 10,9 & 10,4 & 16,4 & 12,9 & 11,3 \\
\hline France & 495 & 505 & 499 & 22,4 & 18,9 & 18,7 & 12,9 & 12,9 & 7,9 \\
\hline
\end{tabular}


Avant d'analyser ce tableau, rappelons que les onze pays ou territoires asiatiques ne sont pas représentatifs du continent asiatique : un pays aussi vaste et peuplé que l'Inde en est absent, tout comme la plupart des pays de culture musulmane ; la Chine n'y est représentée que par des territoires côtiers qui sont par ailleurs des centres commerciaux et financiers de grande importance.

Si l'on examine les scores moyens en mathématiques des pays asiatiques que nous avons rangés par ordre décroissant, deux groupes radicalement différents apparaissent. Un groupe de sept pays ou territoires très performants est composé de Shanghai, Singapour, Hong-Kong, Taïwan, la Corée du Sud, Macao et le Japon, avec des scores nettement supérieurs à la moyenne des pays de l'OCDE et même aux deux pays occidentaux de référence que sont la Finlande et le Canada. Par contre, trois pays asiatiques sont sous-performants avec des scores largement inférieurs à la moyenne de l'OCDE : la Thaïlande, la Malaisie et l'Indonésie (avec des scores particulièrement faibles pour ce dernier). Le Vietnam, pays appartenant à la région sinisée comme les sept pays performants, obtient un score moyen qui ne permet pas (ou pas encore) de le situer dans la première catégorie, malgré un score supérieur à la moyenne de l'OCDE et du même ordre que celui de la Finlande et du Canada.

Cette première tentative de catégorisation se retrouve-t-elle dans les résultats en lecture et en science ? En lecture, on observe à peu près la même configuration, si ce n'est que la Finlande et le Canada obtiennent des scores proches de Taïwan et même supérieurs à ceux de Macao. En ce qui concerne la Finlande, Mark Bray a fait oralement observer que la tradition protestante conduit très tôt les élèves finlandais à la lecture de la Bible. Le bilinguisme précoce des Finlandais et des Canadiens pourrait être un facteur complémentaire de l'explication de ces bons résultats. Pour la culture scientifique, on retrouve la configuration précédente : la Finlande et le Canada, qui attachent une grande importance à la culture technologique, figurent aux côtés des sept pays ou territoires du Sud-Est asiatique. En revanche, la France reste dans la moyenne de l'OCDE pour les trois types de performances et ne parvient pas à se hisser au niveau des résultats du Vietnam.

Si l'on regarde maintenant l'équité de ces systèmes éducatifs, exprimée en termes de pourcentages d'élèves très performants (au-delà du niveau 5) et sous-performants (en deçà du niveau 2, c'est-à-dire ne maîtrisant pas les compétences jugées essentielles), l'on découvre à nouveau que, pour les mathématiques, les élèves des sept pays ou territoires asiatiques ont significativement davantage d'élèves très performants (23,7 \% pour le Japon ; 55,4 \% pour Shanghai) et moins d'élèves sous-performants (12,8\% pour Taïwan ; 3,8 \% pour Shanghai). Si la Finlande et le Canada parviennent à minimiser le pourcentage d'élèves ne maîtrisant pas les compétences de base, ces deux pays ne parviennent pas à obtenir un pourcentage élevé d'élèves très performants (respectivement 16,4 \% et 12,9\%). Pour la lecture et les sciences, la configuration est assez semblable, à l'exception de Macao, qui obtient des résultats similaires à ceux de la Finlande et du Canada. 
Pour les trois types de performances, la France présente des pourcentages assez semblables à la moyenne des pays de l'OCDE et ne parvient guère à faire mieux que le Vietnam, dont les pourcentages d'élèves peu performants sont moins élevés; on peut surtout s'inquiéter de voir la France avec plus d'élèves en réelle difficulté que d'élèves très performants. La Thaïlande, la Malaisie et l'Indonésie, qui avaient des scores d'efficacité peu enviables, ont également des indices de non équité inquiétants : pas d'élèves très performants et énormément d'élèves ne maîtrisant pas les compétences essentielles.

Comme le montrent les travaux de Mons (2007) et de Ghesquière (2013), il est utile de ne pas se contenter des mesures précitées d'équité et de prendre aussi en considération la relation entre les performances et le niveau socioéconomique des familles. Les analyses de l'OCDE (Vayssettes, 2014) montrent alors que si HongKong, Macao et le Vietnam, au même titre que la Finlande et le Canada, ont à la fois des performances élevées et des corrélations plus faibles avec le niveau socioéconomique, ce n'est pas le cas de Shanghai, de Singapour et surtout de Taïwan, dont le niveau de performances est fortement lié au niveau socioéconomique. Avec leurs performances très faibles, la Thaïlande, la Malaisie et l'Indonésie ont de plus des scores d'iniquité élevés exprimés en termes de corrélation avec le niveau socioéconomique.

\section{LES FACES VISIBLES : QUELQUES COVARIATIONS À NE PAS NÉGLIGER}

À ce stade du raisonnement, il nous faut donc distinguer deux grandes catégories de pays asiatiques en fonction de leur efficacité : sept pays ou territoires très performants (Shanghai, Singapour, Hong-Kong, Taïwan, la Corée du Sud, le Japon et Macao) sans doute bientôt rejoints par le Vietnam ; trois pays aux performances très faibles (Thaïlande, Indonésie et Malaisie). Ces derniers sont de plus inéquitables : trop d'élèves ne maîtrisant pas les compétences essentielles; peu ou pas d'élèves très performants ; influence forte du statut socioéconomique. Les sept pays du premier groupe se distinguent de la Finlande et du Canada par un très grand nombre d'élèves très performants et peu d'élèves ne maîtrisant par les compétences essentielles. Cependant, le niveau socioéconomique a une influence encore très marquée pour certains d'entre eux (Taïwan, Singapour et Shanghai). Peut-on associer des caractéristiques objectives à la distinction de ces deux grands groupes de pays?

À l'exception de Singapour, tous les pays du premier groupe (y compris le Vietnam) se situent plus à l'est par rapport aux pays du deuxième groupe et appartiennent à la culture sinisée (c'est le cas aussi de Singapour dont $80 \%$ des habitants sont d'origine chinoise). La culture confucéenne partage un certain nombre de valeurs : importance accordée à l'éducation par les familles et par les autorités éducatives, très grand respect pour le "maître ", qui jouit d'un statut social élevé, valeur de l'effort. Ces pays (Japon, Corée du Sud, Singapour) ou 
territoires (Shanghai, Hong-Kong, Taïwan, Macao) accordent beaucoup d'importance aux développements technologiques et ont des zones portuaires commerciales très importantes.

D'autres indices objectifs ${ }^{2}$ distinguent les deux groupes: les pays ou territoires du premier groupe ont une population rurale moins importante, un PIB par habitant plus élevé, plus de secteurs d'activités consacrées à l'industrie et aux services, des indices de fécondité moins élevés et une population âgée de moins de 14 ans moins nombreuse. Ceci permet de comprendre que les familles du premier groupe de pays peuvent plus facilement supporter les coûts de la scolarité de leurs enfants et que l'espérance de vie scolaire de ces derniers est plus grande que dans les pays du second groupe.

Cependant, d'autres indices objectifs ne distinguent pas les deux groupes de pays, notamment deux : les dépenses publiques totales pour l'éducation en pourcentage du PIB et le ratio élèves/enseignant. Ainsi, des pays comme la Thaïlande et la Malaisie dépensent proportionnellement deux fois plus pour l'éducation que Singapour et Hong-Kong. Par ailleurs, des pays aux performances très différentes ont des ratios élèves/enseignants très comparables, comme le montrent les exemples suivants pour le primaire : Corée du Sud (20), Japon (17), Singapour (17), Indonésie (18), Thaïlande (16). Pays peu efficace et équitable, la Malaisie utilise des ratios particulièrement bas, tant pour le primaire que pour le secondaire (respectivement 12 et 14). Puisqu'il convient de se méfier d'indices trop synthétiques, il faut sans doute chercher d'autres pistes d'explication.

L'une de ces pistes est à rechercher dans les travaux récents de Ghesquières (2013) qui, avec Nathalie Mons (2007), distingue quatre groupes de pays selon la manière dont sont traités les élèves en difficulté scolaire dans l'enseignement primaire et secondaire :

- le modèle de la séparation, où les élèves sont séparés selon leur niveau académique ; le tronc commun est court, des classes de niveau peuvent être mises en place dès le primaire et, dans le secondaire, les élèves sont répartis en filières hiérarchisées ; le taux de redoublement y est plutôt élevé ;

- le modèle de l'intégration uniforme, où la pratique du redoublement est intensive mais où les filières n'ont pas d'existence précoce, où les pratiques de répartition par niveau sont peu fréquentes et où l'aide individualisée est rare ;

- le modèle de l'intégration à la carte, où les élèves redoublent peu et ne sont pas orientés vers des filières différentes, mais où les pratiques de répartition par niveau sont plus fréquentes et où les élèves suivent des enseignements à la carte dans une filière unique ;

- le modèle de l'intégration individualisée, où les élèves ne redoublent pas, ne sont pas orientés dans des filières différentes et où les pratiques de répartition par niveau sont peu répandues, mais où les aides individualisées sont fréquentes.

2. On peut retrouver les données chiffrées dans l'important dossier documentaire réalisé pour le colloque par Céline Brun, du centre de ressources et d'ingénierie documentaires du CIEP. Les chiffres fournis correspondent à des données établies en 2012 et 2013 (Brun, 2014). 
En prenant en considération ces quatre modèles, Ghesquière fournit les résultats de 28 pays sur la base de trois mesures : l'inégalité des chances en termes de corrélation entre l'indice PISA 2006 du statut social, économique et culturel d'une part, et les performances en mathématiques et en lecture d'autre part ; l'inégalité des résultats mesurée par l'écart-type des compétences des élèves en mathématiques et en lecture ; l'inégalité des acquis de base exprimée en pourcentages d'élèves qui n'ont pas atteint le niveau 2 dans les deux domaines précités. Dans ces 28 pays analysés, nous retrouvons cinq des pays présents dans le tableau 1. Notons que nous ne retrouvons aucun pays asiatique précité dans le modèle de la séparation, ce qui nous a conduit à prendre en considération des mesures moyennes pour les dix pays pris en considération par l'auteur. Dans le tableau 2, nous avons sélectionné les résultats intéressants pour notre démonstration.

Tableau 2.

Croisement entre quatre modèles de politiques éducatives et trois mesures d'inégalité (PISA 2006, mathématique et lecture)

\begin{tabular}{|c|c|c|c|c|c|c|}
\hline \multirow{2}{*}{ Modèle } & \multicolumn{2}{|c|}{ Inégalité des chances } & \multicolumn{2}{|c|}{ Inégalité des résultats } & \multicolumn{2}{|c|}{$\begin{array}{c}\text { Inégalité des acquis } \\
\text { de base }\end{array}$} \\
\hline & Maths & Lecture & Maths & Lecture & Maths & Lecture \\
\hline \multicolumn{7}{|l|}{ Séparation } \\
\hline Moyenne des pays & 0,411 & 0,389 & 95,3 & 102,4 & $18,4 \%$ & $20,2 \%$ \\
\hline \multicolumn{7}{|c|}{ Intégration uniforme } \\
\hline France & 0,462 & 0,405 & 95,6 & 104,0 & $22,5 \%$ & $21,9 \%$ \\
\hline \multicolumn{7}{|c|}{ Intégration à la carte } \\
\hline Canada & 0,281 & 0,309 & 85,8 & 96,3 & $10,9 \%$ & $11,1 \%$ \\
\hline \multicolumn{7}{|c|}{ Intégration individualisée } \\
\hline Corée du Sud & 0,331 & 0,257 & 92,6 & 88,3 & $8,9 \%$ & $5,9 \%$ \\
\hline Finlande & 0,316 & 0,278 & 80,9 & 81,2 & $6,0 \%$ & $4,9 \%$ \\
\hline Japon & 0,307 & 0,271 & 91,0 & 102,4 & $13,2 \%$ & $18,6 \%$ \\
\hline
\end{tabular}

Source : repris de Ghesquière (2013).

Les résultats du tableau 2 montrent clairement que les modèles d'intégration à la carte et individualisée sont moins inégalitaires (corrélations, écartstypes et pourcentages plus faibles) que les modèle de la séparation et de l'intégration uniforme: peu ou pas de redoublements, pas de filières hiérarchisées, enseignement à la carte ou pratiques individualisées, autant d'éléments qui réduisent les inégalités et ne sont pas incompatibles avec de hauts niveaux de performances. Ceci rejoint l'idée forte de l'Unesco, selon laquelle efficacité et équité sont inséparables. Ces aspects méritent d'être étudiés en prenant l'ensemble des pays asiatiques ayant participé aux enquêtes PISA 2012. 


\section{LES CARACTÉRISTIQUES NON VISIBLES DES ENQUÊTES INTERNATIONALES}

Dans un article (De Ketele, 2010), nous insistions sur l'importance d'analyser les classements internationaux en utilisant une série de questions à double face, afin d'éviter des inférences abusives.

Qui conçoit? Qui ne conçoit pas? La question se pose ici en termes de commanditaire coordinateur du PISA, cette vaste opération, et des acteurs clés grâce auxquels elle se réalise. Il s'agit en premier lieu de l'OCDE, l'Organisation de coopération pour le développement économique, grand défenseur d'une "économie de la connaissance ». Cette organisation recourt à des experts qui, pour la plupart, sont des spécialistes de la mesure en éducation (des psychométriciens, constitués en équipes internationales et nationales). Il s'agit d'une catégorie restreinte d'acteurs, qui ne comprend pas les experts de terrain du système éducatif que sont les enseignants, les parents, les responsables des politiques éducatives, les associations qui réfléchissent aux différents aspects d'un développement humain et social durable. Le dispositif est donc laissé aux mains d'une seule catégorie d'experts, par ailleurs très compétents.

Qu'est-ce qui est évalué? Qu'est-ce qui n'est pas évalué? L'OCDE a le mérite de dire clairement qu'il ne s'agit pas d'évaluer les acquis scolaires, tant ils peuvent différer d'un système à l'autre et tant l'élaboration d'un plus petit dénominateur commun est difficile. Il s'agit plutôt d'évaluer les «compétences » nécessaires à tout jeune de quinze ans pour entrer dans une société de plus en plus complexe et globalisée. Ces compétences seraient donc une certaine culture liée à la lecture, la mathématique et la science. Au-delà de la question de la définition de ce qui caractérise ces cultures se pose évidemment la question de savoir si d'autres aspects ne sont pas également (ou tout autant) essentiels pour vivre en société, et par exemple les aspects culturels liés aux histoires et aux contextes locaux qui constituent les identités; les aspects liés à la citoyenneté et aux enjeux posés par la société... Autant d'aspects qui sont plus difficilement mesurables.

Fonctions recherchées? Fonctions non recherchées? La fonction la plus visiblement utilisée et largement médiatisée est sans conteste la fonction de classement ou de palmarès. Cette fonction domine de fait la fonction proclamée de régulation des systèmes éducatifs. Certes, certains pays (en Europe, l'Allemagne est sans doute l'un des exemples les plus frappants) ont profité du choc de la proclamation des résultats pour entreprendre des réformes profondes. Mais trop souvent, il s'agit d'une volonté de mieux se positionner dans les classements (d'améliorer le score moyen) et donc de se conformer le plus étroitement possible aux normes sous-jacentes des enquêtes (par le bachotage notamment), en oubliant souvent les aspects non évalués ou en négligeant les aspects liés à l'équité. La régulation reste trop souvent globale et les acteurs locaux se sentent peu concernés ou dénoncent la fonction de déculturation de ces classements internationaux. 
Au profit de qui? Au détriment de qui ? Les destinataires principaux des résultats PISA sont les autorités internationales et nationales, principalement celles qui sont persuadées du lien entre éducation et développement économique. Elles ont également un écho important auprès des médias, qui y voient l'occasion de «faire la une » pendant une période limitée. Elles trouvent très peu d'écho auprès des acteurs locaux, puisque les résultats restent globaux et ne sont pas disséqués par établissement.

Procédures mises en œuvre? Procédures évitées? Les épreuves internationales, et de plus en plus souvent les épreuves nationales lorsqu'elles existent, recourent désormais à un modèle de validation des épreuves, la théorie de la réponse à l'item (TRI), qui se fonde sur plusieurs postulats, dont le principal est l'unidimensionnalité de l'objet évalué. Or les tâches complexes qui caractérisent les compétences les plus pertinentes sont de nature multidimensionnelle et ne peuvent se réduire à un score global unique. De même, ce modèle de validation tend à rejeter ou minimiser tout ce qui est appelé "biais culturels ». Les procédures des épreuves évitent donc les tâches complexes ou les décomposent en tâches plus simples; elles tendent aussi à neutraliser tout enracinement culturel, comme si cela était possible (la plupart des items sont d'abord formulés en anglais, puis traduits ; or on sait le lien étroit entre la langue et la pensée). En un mot : elles subordonnent l'objet à évaluer au modèle de validation.

Paradigmes mis en cuvre? Paradigmes évités ? À partir de ce qui vient d'être dit, on peut se demander si le paradigme prioritaire sous-jacent aux épreuves PISA ne consiste pas à développer " un seul monde et une seule école » (thème du précédent colloque international organisé par la Revue internationale d'éducation de Sèvres en 2009), les deux servant au développement d'une "société de la connaissance » et même d'une "économie de la connaissance ", se pliant à des "standards internationaux » édictés par des experts à l'autorité reconnue, auxquels il conviendrait de se conformer et en fonction desquels les classements sont établis. Or « qui classe déclasse » et contribue à mettre en avant une « excellence élitiste » que seuls quelques systèmes éducatifs atteindront. Ne pourrait-on, au contraire, viser prioritairement un autre paradigme, à savoir celui d'une école riche de sa diversité et d'une excellence sociale (éducation de qualité pour tous, qui permet à chacun de mobiliser son potentiel et d'atteindre ainsi son propre niveau d'excellence) et sociétale (éducation sensible aux enjeux locaux, nationaux et internationaux posés par la vie en société et son devenir).

Effets indirects positifs? Effets indirects pervers? Sans conteste, les épreuves internationales ont eu des effets indirects positifs. On ne peut nier qu'elles évaluent des aspects importants et que la médiatisation des résultats a amené les autorités politiques et éducatives à se poser la question du bien-fondé de leur politique éducative, non seulement celle qui est prescrite mais aussi celle réellement mise en œuvre. Si, comme l'affirment les comparatistes, la compréhension d'un système passe par la comparaison avec d'autres systèmes - fondement 
de l'éducation comparée (Bray, 2010) -, alors les enquêtes internationales, surtout lorsqu'elles sont répétées dans le temps (c'est le cas de PISA), sont un puissant outil pour les pays ou territoires qui prennent la peine d'étudier en profondeur les informations récoltées par les enquêtes complémentaires adressées aux chefs d'établissement et qui ajoutent d'autres études plus fines et contextualisées. Mais les effets pervers ne sont pas à négliger. Le principal est l'effet de " déclassement " induit par le classement, surtout quand il s'accompagne d'une vision trop étroite de la signification des résultats obtenus : attention trop exclusive aux faces directement visibles, déductions abusives trop rapides, absence des contextualisations nécessaires, oubli de comparer ce qui est comparable. D’autres effets conjoints ont déjà été mis en évidence : la valorisation de la compétition au détriment de la coopération; le bachotage sur la base des épreuves antérieures au détriment d'une approche en profondeur et contextualisée.

Quel type de développement personnel est privilégié? Quel type de personne n'est pas privilégié ? En mettant l'accent sur l'acquisition d'un capital de connaissances et de compétences pour permettre à la personne de vivre dans une société de plus en plus complexe, mobile, compétitive et où le développement économique est l'étalon principal, ne court-on pas à terme vers certaines catastrophes comme le laissent entendre tous ceux qui étudient les problématiques liés au développement humain : l'avoir au détriment de l'être ; dominants et dominés ; identités uniformes au détriment des identités riches de leur diversité ?

Quel type de société est privilégié? Pour retarder l'émergence de quel type de société ? En mettant l'accent sur l' «homo economicus", ne renforçons-nous pas une société à plusieurs vitesses puisque les classements et la compétition qu'ils engendrent mettront toujours en évidence quelques systèmes sociaux et éducatifs en tête et d'autres à la traîne ? L'économie de la connaissance ne sertelle pas l'intérêt de quelques-uns (de plus en plus une oligarchie financière) au détriment du bien-être des autres, comme le montrent les fossés de plus en plus grands entre les régions du monde ou, à l'intérieur des pays ou territoires euxmêmes, entre des classes sociales aux capitaux sociaux et économiques extrêmement différents ?

Cette brève analyse, conduite de façon générale et sans allusion directe aux systèmes éducatifs et aux sociétés asiatiques, nous paraît indispensable pour aborder de façon plus distanciée, compréhensive et non réductionniste les résultats livrés par les enquêtes PISA. Est-il étonnant que les sept pays ou territoires asiatiques les plus performants sur les objets évalués par les enquêtes soient situés dans une même région (de culture sinisée) et soient tous en plein développement économique, tournés vers le commerce mondial ? Aurait-on eu des résultats semblables dans d'autres territoires non côtiers de la grande Chine ? Les performances comparativement plus faibles du Vietnam ne sont-elles pas partiellement explicables par les nombreuses ethnies vivant dans les montagnes reculées de ce pays? La diversité culturelle, langagière et ethnique n'explique-t-elle pas, en 
partie du moins, les faibles niveaux de performances de la Thaïlande, la Malaisie et l'Indonésie? Les objets évalués par les épreuves PISA sont-ils les plus pertinents pour de tels pays ? Ne faudrait-il pas mettre en évidence d'autres acquis et valeurs qui leur sont propres et que l'on ne retrouverait pas nécessairement dans les pays aux classements favorables? Ne faudrait-il pas prendre en compte d'autres facettes, souvent occultées pour l'instant, pour comprendre en profondeur la diversité des systèmes éducatifs et la qualité de leurs performances?

\section{Des faCes OCCultées À PRENDRE EN CONSIDÉRATION}

L'enquête PISA fournit des résultats précieux, comme nous l'avons dit. L'un de ses principaux mérites est d'englober dans la qualité deux concepts indissociables : l'efficacité (le rapport entre les résultats observés et les résultats attendus) et l'équité (la distribution juste des résultats observés selon les sousgroupes de population). Si l'on retrouve bien, dans les publications de l'OCDE, tous les résultats en termes d'efficacité et d'équité, on doit cependant constater que, dans le processus de communication, ce sont les résultats en termes d'efficacité qui sont mis en évidence et largement diffusés sur le plan médiatique, et non les résultats en termes d'équité. Ne serait-il pas aussi important de mettre en évidence ces derniers, très largement et au moins de façon aussi importante, en prenant en compte les trois mesures précitées : la corrélation avec le statut socio-économique des familles; l'écart-type des performances (traduisant leur dispersion) ; le pourcentage d'élèves ne maîtrisant pas les compétences essentielles ? Nous aurions ainsi, pour chacun des objets évalués, quatre mesures, publiées côte à côte et qui donneraient une image plus juste d'une certaine vision de la qualité des systèmes éducatifs.

Nous employons volontairement l'adjectif indéfini "certaine » pour exprimer le caractère relatif de cette vision. Car nous pensons que la vision de la qualité ne peut se réduire à la seule évaluation des performances d'une part, mais doit prendre en compte, en termes systémiques, la qualité des inputs et des facteurs processuels d'autre part, sans omettre de considérer la pertinence de ce que les élèves doivent apprendre (résultats attendus), ce qui est appris réellement et enfin ce qui est évalué.

Le concept de «pertinence » est un concept trop souvent négligé ; il est cependant une composante essentielle de la qualité au même titre que l'efficacité et l'équité. Il désigne le degré d'adéquation entre les besoins sociaux et sociétaux d'un environnement donné et les résultats attendus (prescrits), appris réellement et évalués. Si de grands pays asiatiques, tels que le Japon et la Corée du Sud, et des territoires nettement plus restreints, comme Shanghai, Singapour, HongKong, Taïwan et Macao, sont des sociétés complexes et relativement homogènes, il n'en va pas de même pour la Thaïlande, la Malaisie et l'Indonésie. Ces pays 
très vastes sont composés de plusieurs sociétés hétérogènes, tantôt rurales et tantôt urbaines, aux ressources et aux habitudes de vie très différentes, aux langues, valeurs et cultures multiples. Ces pays sont donc composés de territoires forts différents et aux besoins prioritaires non semblables. C'est partiellement encore le cas d'un pays comme le Vietnam où certains territoires reculés dans les montagnes sont composés d'ethnies diverses, dont certaines vivent en relative autarcie et avec des modes vie qui ont peu à voir avec les sociétés modernes actuelles. On peut donc déduire de cette analyse que les objets évalués par PISA sont plus pertinents pour le premier groupe de pays et de territoires, mais nettement moins pour d'autres territoires ou pays asiatiques. Chez ces derniers, nous retrouverions sans doute des besoins prioritaires liés aux besoins de survie, de coopération, de solidarité, de transmission des savoirs d'expérience... La comparaison ne prend sens que si l'on compare des objets comparables.

Nul ne contestera que pour évaluer la qualité, il est nécessaire de prendre en compte les résultats de questionnaires d'évaluation bien construits (et ils le sont dans le cas des épreuves PISA). Si les objets évalués ne sont pas le fruit d'un plus petit commun dénominateur des programmes scolaires, ils n'en sont pas moins peu éloignés et tendent même à devenir progressivement, et pour une grande part, le "curriculum prescrit » de bien des systèmes éducatifs. À côté de ce curriculum prescrit coexiste un " curriculum caché », dont les effets sont aussi importants et dont les experts disent qu'il laisse, à long terme, plus de traces que le curriculum prescrit, car il agit profondément sur les " postures » des élèves, en induisant des comportements qui sont ensuite intériorisés par le futur adulte. Les caractéristiques de l'environnement dans lequel s'inscrivent l'acte éducatif et l'acte d'enseignement/d'apprentissage jouent un rôle déterminant sur le devenir de l'élève. Elles sont donc à prendre en compte dans l'évaluation de la qualité d'un système éducatif.

Dans son programme "Régulation par l'État de la qualité et de l'équité de l'éducation » (De Ketele, 2014), l'Organisation internationale de la francophonie l'a bien compris en prenant en compte les composantes suivantes de la qualité :

- outre l'accès (composante de l'équité), l'accessibilité : l'existence des établissements et des personnels ; les différents dispositifs facilitateurs (proximité, transports, gratuité effective, soutiens sociaux...);

- la qualité des ressources mises à disposition : équipements et maintenance ; financements et appuis financiers ; personnels formés et accompagnés ; supports pédagogiques; environnement scolaire participant de l'éducation ; gestion et fonctionnement des établissements ; existence, diffusion et application des textes;

- la qualité des performances scolaires et des aspects éducatifs : les indicateurs quantitatifs (taux de réussite, d'échec, de redoublement, d'abandon, d'achèvement, de promotion) qui, ne l'oublions pas, ont des effets importants 
sur l'indispensable confiance en soi des élèves; les indicateurs qualitatifs (le type de performances évaluées, les différents effets éducatifs induits); les progrès réalisés par une même cohorte dans une période et un environnement donnés ;

- la qualité en termes de pertinence du curriculum prescrit et réel : pertinence de ce qui est prescrit comme devant être enseigné et appris ; pertinence de ce qui est effectivement enseigné et appris ; pertinence du curriculum caché.

Même si les enquêtes complémentaires, dans les dispositifs PISA, nous fournissent des informations intéressantes sur certaines de ces composantes de la qualité (par exemple, les questions sur le climat de la discipline dans les écoles, le plaisir d'apprendre des élèves, leur anxiété, leur confiance en soi, leur persévérance, les dispositifs d'amélioration et d'assurance qualité...), nous disposons encore de trop peu d'informations. Par ailleurs, celles-ci sont trop souvent agglomérées dans des indices globaux ne tenant pas compte des besoins territoriaux ou des sous-groupes de population, et sont encore trop peu connues et exploitées. Sans doute, nous aurions été en mesure de mieux comprendre (au sens étymologique du terme) pourquoi les différents systèmes éducatifs asiatiques évalués par PISA se subdivisent en plusieurs groupes de pays et de territoires et en quoi certains d'entre eux pourraient inspirer nos propres pays.

\section{BIBLIOGRAPHIE}

BRAY M., ADAMSON, MASON M. (2010): Recherche comparative en éducation. Approches et méthodes, Bruxelles : De Boeck.

BRUN C. (2014): Sélection de données statistiques, colloque: "L'éducation en Asie en 2014 : quels enjeux mondiaux?", CIEP, 12-14 juin 2014. [http://goo.gl/GnlFMf]

DE KETELE J.-M. (2014) : «Régulation étatique, qualité et équité en éducation : une approche originale de six pays francophones ", Revue internationale d'éducation de Sèvres, $\mathrm{n}^{\circ} 65$, p. 23-28.

DE KETELE J.-M. (2010) : «Faces visibles et cachées des classements internationaux. Une tentative de modélisation des tensions dans l'enseignement obligatoire ", Revue internationale d'éducation de Sèvres, $\mathrm{n}^{\circ}$ 54, 2010, p. 39-49 [en ligne] http://ries.revues. org/849

GHESQUIERE F. (2013) : "Les différentes conceptions de l'égalité scolaire à l'épreuve de l'enquête PISA 2006 », Revue française de pédagogie, n 185, p. 69-84.

MONS N. (2007): Les nouvelles politiques éducatives: la France fait-elle les bons choix? Paris : PUF.

Revue internationale d'éducation de Sèvres (2009): "Un seul monde, une seule école? ", dossier, $n^{\circ} 52$, décembre.

VAYSSETTES S. (2014) : Évaluer les systèmes pour améliorer l'éducation, conférence donnée au Collège des Bernardins, Paris, 20 octobre. 